\title{
Triglycerides-to-HDLC Ratio as a Marker of Cardiac Disease and Vascular Risk Factors in Adults
}

\author{
Qurat ul Ain, Naveed Asif, Amir Alam, Mehwish Gilani, Noreen Shahzad and Waqas Sheikh \\ Department of Chemical Pathology \& Endocrinology, Armed Forces Institute of Pathology (AFIP), Rawalpindi, Pakistan
}

\begin{abstract}
Objective: To find out correlation of triglycerides-to-HDL cholesterol ratio in elders with cardiac risk factors like obesity, diabetes, and hypertension.

Study Design: Observational cross-sectional study.

Place and Duration of Study: Department of Chemical Pathology and Endocrinology, Combined Military Hospital, Rawalpindi, from January to June 2018.

Methodology: Inclusion standards were participants aged 20-59 years .Patients with comorbidity and chronic illness were excluded from the study. A planned standardised homogeneous survey was conducted as a pilot study. Social, economic and physical variables like age, gender, marital status and presence of diseases, all were taken into consideration. To check association among cardio metabolic risk factors like diabetes, obesity, hypertension, and TG/HDL-C ratio, Chi-square test was computed for all cardio metabolic risk factors.

Results: Overall 350 participants were studied after attainment of authorisation from Ethical Review Board of Combined Military Hospital, Rawalpindi, in which $268(76.6 \%)$ were women; whereas, $82(23.4 \%)$ were men with mean age of 37 \pm 11.64 years. There was strong association of TG/HDL ratio with BMI, HOMA-IR, WBISI, visceral fats, smooth muscle mass, HDL, LDL, and triglycerides with $p<0.04, p<0.001, p<0.01$, and $p<0.001$, respectively.

Conclusion: TG/HDLC ratio can be considered as a potential biomarker for the early prediction of cardiometablic risk factors.
\end{abstract}

Key Words: High density lipoprotein, Cardiovascular disease, Lipids ratio, Obesity.

How to cite this article: Ain QU, Asif N, Alam A, Gilani M, Shahzad N, Sheikh W. Triglycerides-to-HDLC ratio as a marker of cardiac disease and vascular risk factors in adults. J Coll Physicians Surg Pak 2019; 29(11):1034-7.

\section{INTRODUCTION}

Cardiovascular diseases (CVD) are the leading cause of mortality, almost accounting for 17.7 million deaths globally, which is almost $30 \%$ of global deaths. ${ }^{1}$ The most common cause of cardiac diseases is ischemic disease, rheumatic heart disease. Four out of five cerebrovascular diseases are due to cardiac ischemic attack and strokes .Among these, 32\% females while $27 \%$ of males died due to CVD in $2004 .^{2}$ Around $80 \%$ of all CVD deaths are due to heart attack and stroke. CVD is the leading cause of death worldwide and it causes $47 \%$ of all deaths in Europe. Most of cardiovascular deaths are caused by coronary heart disease (CHD), which is the only most common reason of death in Europe and the United States. ${ }^{3}$

Many efforts have been made to decrease the prevalence of $\mathrm{CHD}$ through modifications in lifestyle and early medical intervention; and as a result, CHD mortality has fallen in many European countries during the last

Correspondence to: Dr. Qurat ul Ain, Department of Chemical Pathology and Endocrinology, AFIP, Rawalpindi, Pakistan

E-mail: draaneiseera@gmail.com

Received: January 09, 2019; Revised: July 15, 2019;

Accepted: August 26, 2019 decades. However CHD is still the main cause of premature death in Europe accounting for about $30 \%$ of deaths before the age of 65 years. ${ }^{4}$ There is, therefore, a need for more research on CHD to allow for more effective preventive measures. Atherosclerosis is the predominant cause of CVD, especially CHD. According to current knowledge, most acute CHD events are caused by rupture of plaques with relatively high lipid content and thin fibrous cap, called unstable plaques. It is predictable that $54 \%$ non-communicable diseases occur in Eastern Mediterranean Region (EMRO) and most common is CVD. Mortality rate due to cardiac disease is $49 \%$ in Oman, while $13 \%$ in Somalia. 5 The prevalence of CVD is dependent on sedentary lifestyles, increase in blood pressure $(28 \%$ in the United Arab Emirates to $41 \%$ in Libya and Morocco); while diabetes (4\% in Islamic Republic of Iran to $19 \%$ in Sudan) and dyslipidemia (14\% in Lebanon to $52 \%$ in Islamic Republic of Iran). 6

Triglyceride-to-HDL-Cholesterol ratio is a good predictive marker for insulin resistance and for early assessment of CVD. In some studies, TG-to-HDLC ratio less than 0.87 is considered ideal, value above $>1.74$ is too high and high risk for coronary artery disease. In one study TG/HDL Cholesterol ratio is considered self-governing 
predictable factor for coronary artery disease. ${ }^{7}$ There is substantiation that ratio of "triglyceride (TG)/ and high density cholesterol (HDL-C)" makes available a meek technique to recognise outwardly healthy insulin resistant persons with augmented cardiac and metabolic threat dynamics.

Additional studies proved that it has been revealed that the most useful TG/HDL-Cholesterol cut-off point to identify cardiac and metabolic threat are different in both male and female. ${ }^{8}$ Latest studies have shown that this ratio is a proven prognostic factor for progress of $\mathrm{CHD}$ and is associated with plasma; B-type 'LDL' cholesterol levels. ${ }^{9}$ Therefore, recent study was conducted to analyse 'TG-to-HDL cholesterol ratio' in hospital settings as a predictor for early CVD factors.

\section{METHODOLOGY}

This cross-sectional study was initiated after Institutional Review Board's (IRB) approval at CMH Rawalpindi from January to June 2018. Data was composed from 350 patients who visited AFIP. Samples were obtained after informed consent. The Research Ethics Committee of AFIP approved the study protocol. Patient with the age group 20-59 years were included in the study, while participants with comorbidities like tuberculosis, tumour or any other chronic illness were excluded from the study.

Sampling technique was non-probability convenient sampling. Questionnaire was validated before conducting study as pilot project. The self-determining factors assessed in initial meeting were age and other demographic features as well as presence of chronic diseases. In second meeting, weight and height with BMI were calculated for obesity classification (weight, height and waist circumference were gauged). We have categorised three main groups for BMI as defined by World Health Organization (Normal, overweight and obese).

Participants with $\mathrm{BMI}<25$ were measured normal, whereas BMI of 25.5-30 was considered overweight and more than 30 was considered as obese. Biochemical assessment was performed fasting overnight for 10-12 hours, with plasma glucose, lipid profile (total cholesterol, HDL-C, LDL-C, VLDL-C and triglycerides), and fasting insulin for Insulin-resistance parameter like (HOMA IR-Quicki). Blood sample was taken from selected participants after 10-12 hour fasting through venipuncture in gel tube (yellow topped) for lipid profile, fasting glucose sample was collected in sodium fluoride tube and was immediately sent to laboratory for analysis (Centrifugation and analysis). Sample for lipid profile concentration and fasting glucose was analysed on fully automated chemistry analyser, while serum insulin fasting was analysed on fully automated instrument based on chemilumiscese principle.

Triglycerides to HDL Cholesterol ratio is calculated by triglycerides value divided by HDL-Cholesterol value.
HOMA-IR was calculated by multiplying fasting plasma glucose value with serum insulin fasting divided by 22.5 , while WBISI and QUICKI were measured and calculated manually. Data was analysed using SPSS Version 24. For descriptive stastics, mean $\pm S D$ computed for quantitave variables, while frequency was calculated for qualitative variables. Chi-square test was applied for association, and one-way Anova computed for all variables to prove TG/HDL-C ratio association with all risk factors for early prediction by considering significant association at $p$-value less than 0.05 .

\section{RESULTS}

Overall, 350 participants was studied in which 268 $(76.6 \%)$ were females and $82(23.4 \%)$ were males with mean age of $37 \pm 11.64$ years. Sixty $(17 \%)$ were with BMI 18.5-25 and considered as normal, while 192 (55\%) were with BMI 25-30. There were $278(79.4 \%)$ participants who belonged to urban area, while $72(20.6 \%)$ belonged to rural area. There were $203(58 \%)$ patients diagnosed previously with primary hypertension, while 147 (42\%) had no hypertension and considered healthy. Most of the patients with diagnosis of hypertension had duration of 2-3 years of disease. There were $195(55.7 \%)$ participants already diagnosed with diabetes on the basis of $\mathrm{HbA} 1 \mathrm{c}$ and oral glucose tolerance test, while 155 (44.3\%) were non-diabetic. Patients with CHD diagnosed with angiography were $63(18 \%)$, while those who did not have CHD were 287 (82\%).

\begin{tabular}{l|c|c}
\multicolumn{3}{|c}{ Table I: Descriptive stastics of quantitative variables ${ }^{*}$ In male and female). } \\
\hline Variables & Male (Mean \pm SD) & Female (Mean \pm SD) \\
\hline Age & $38 \pm 11.0$ & $37 \pm 13.2$ \\
Height(cm) & $170.1 \pm 3.64$ & $160.3 \pm 6.22$ \\
Weight(Kg) & $85.3 \pm 15.6$ & $82.3 \pm 11.5$ \\
BMI & $29.4 \pm 4.9$ & $31.5 \pm 15.6$ \\
Visceral fats & $14.0 \pm 3.8$ & $18.9 \pm 1.96$ \\
Skeletal muscle mass & $29.9 \pm 4.3$ & $23.8 \pm 2.4$ \\
Triglycerides (mmol/l) & $1.47 \pm 0.52$ & $1.44 \pm 1.2$ \\
LDL-C (mmol/I) & $2.25 \pm 1.48$ & $2.38 \pm 0.50$ \\
HDL-C (mmol/) & $1.2 \pm 0.66$ & $1.0 \pm 0.23$ \\
Insulin Fasting (IU/ml) & $12.9 \pm 6.5$ & $21.0 \pm 9.1$ \\
HOMA-IR & $0.92 \pm 1.61$ & $2.0 \pm 2.42$ \\
QUICKI & $0.70 \pm 0.45$ & $0.53 \pm 0.61$ \\
TG/HDL Ratio & $1.41 \pm 0.71$ & $1.42 \pm 1.45$ \\
WBISI & $3.47 \pm 2.48$ & $1.41 \pm 0.88$ \\
\hline
\end{tabular}

Table II: One way Anova in three tertiles of TG/HDL-C ratio.

\begin{tabular}{l|c|c|c|c}
\hline Variables & $\begin{array}{c}\text { Tertile1 } \\
(0.1-3.59)\end{array}$ & $\begin{array}{c}\text { Tertile 2 } \\
(3.60-7.18)\end{array}$ & $\begin{array}{c}\text { Tertile 3 } \\
(7.19-10.3)\end{array}$ & $\begin{array}{c}\text { p-value } \\
(0.05)\end{array}$ \\
\hline Age & $37.7 \pm 11.59$ & $33.50 \pm 8.40$ & $28 \pm 5.58$ & 0.700 \\
Weight* & $83.7 \pm 12.57$ & $70.3 \pm 7.18$ & $68.4 \pm 0.10$ & 0.000 \\
BMI & $31.39 \pm 14.09$ & $24.7 \pm 4.74$ & $24.0 \pm 4.6$ & 0.140 \\
Visceral fats* & $17.7 \pm 3.17$ & $19.57 \pm 1.60$ & $20 \pm 0.01$ & 0.050 \\
SMM $^{*}$ & $25.5 \pm 3.96$ & $21.8 \pm 1.17$ & $21.5 \pm 0.012$ & 0.001 \\
HOMA-IR* $^{\text {WBISI }}$ & $1.48 \pm 2.0$ & $6.69 \pm 1.03$ & $7.1 \pm 0.041$ & 0.000 \\
\hline
\end{tabular}




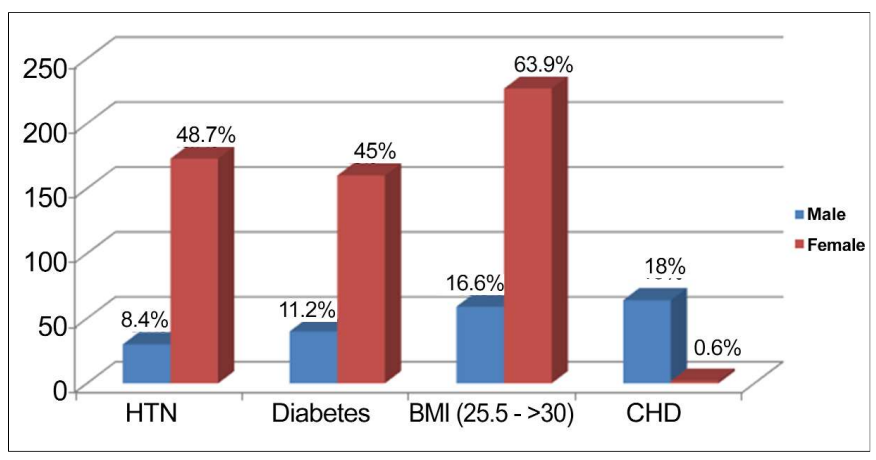

Figure 1: Gender-wise frequency of cardiovascular risk*.

Descriptive stastics for quantitave variables such as mean \pm standard deviation (SD) of all continuous and discrete variables such as age, height, weight, BMI, percent body fats, visceral fats, LDL-C, HDL-C, triglycerides, HOMA-IR, QUICKI, WBISI, and TG/HDL-C ratio with detail to men and women is computed in Table I. Frequency of CHD, hypertension and diabetes is shown in Figure 1. TG/HDL-C ratio was divided in to three tertiles (0.1-3.59, 3.60-7.58, 7.19-10.3). Significance levels with these three tertiles TG/HDL-c ratio with all variables were checked by one-way Anova (Table II). A significant and positive correlation existed between insulin resistance and visceral fats with TG tertiles $(r=0.471, p<0.001, r=1.23, p=0.02)$ and negative association existed between body mass index and TG tertiles $(r=0.97, p=0.05)$. To check association of cardio metabolic risk factors like diabetes, obesity and hypertension with TG/HDL-C ratio, Chi-square test was computed and significant association was showed with all variables except WBSI to ascertain association between TG/HDL-C ratio with all risk factors having strong association among HOMA-IR, diabetes, hypertension, percent body fats and TG/HDL-C ratio $p<0.04$, $p<0.001, p<0.01, p<0.001$, respectively.

\section{DISCUSSION}

This study was conducted with the aim to evaluate significant relationship of TG/HDL-cholesterol ratio with cardiovascular risk dynamics including hypertension, diabetes, and obesity. High value of total cholesterol and triglycerides level can help predict cardiac risk factors. For adults, there is growing interest by clinicians to consider TG/HDL-C ratio as an early predictor to evaluate cardiac risk dynamics. 10 In a study conducted at school-level by Musso et al.11 it is stated that TG/HDLC ratio and CRP positively correlate with high $\mathrm{BMI}$; and these findings correlate with current study findings. Substantiation recommended in a study conducted by Cui et al. ${ }^{12}$ that non-HDL cholesterol is more atherogenic. In this study, results correlate with these findings that higher LDL cholesterol level and higher triglycerides have more risk of developing cardiac disease. A large multi-cohort study by Giannini et al.13 suggested that TG/HDLC ratio correlates well with insulin resistance in girls, while present study revealed that there is a significant positive association between HOMA-IR, a marker used to asses insulin resistance. ${ }^{13}$

A study conducted by Marcus et al. revealed the fact that poor dietary habits lead to cardiovascular complications induced by obesity, which also correlate with the findings of current study that obesity has strong association with cardiovascular risk factors like hypertension and diabetes. ${ }^{14}$ A substitute possibility, with very hopeful findings conducted by Gotto and Whitney et al., 15 stated that use of lipid ratios, just as LDL-cholesterol/HDLcholesterol ratio and triglycerides/HDL-cholesterol ratio is easy to use and sensitive enough to predict cardiac risk factors, which is also evident from results of current study that patients with a normal lipid profile but with a higher value of triglycerides/HDL-cholesterol ratio had a higher BMI and higher visceral fats. ${ }^{16}$ Recent study is first time piloted in the Pakistani population to asses TG/HDL-C ratio as a potential marker to see association of different cardiac risk factors and TG/HDL-c ratio. The limitations are that this is a cross-sectional study and is not enough to express causal association between TG/HDLC ratio with BMI, visceral fats, hypertension, diabetes and insulin resistance, that is why an advanced study design is required to follow-up patients with high TG/HDL-c ratio to prove the fact of positive association with cardiac risk factors. Current study is conducted in a hospital setting, that is why findings cannot be applicable to general population. Large multi-center population based advance research design is required to assess temporal correlation of $\mathrm{TG} / \mathrm{HDL}-\mathrm{c}$ ratio with cardiac risk factors.

\section{CONCLUSION}

This study concluded that there is a strong association of TG/HDLC ratio with body mass index, visceral fats, insulin resistance, hypertension and diabetes. TG/HDLC ratio is considered as potential biomarker for the early prediction of cardiometablic risk factors.

\section{ETHICAL APPROVAL:}

Ethical approval was taken from Institutional Review Board (IRB) of Armed Forces Institute of Pathology (AFIP) prior to start of research.

\section{PATIENTS' CONSENT:}

Informed consents were taken from participants for publishing of research work.

\section{CONFLICT OF INTEREST:}

The authors declare that there is no conflict of interest that could be perceived as prejudicing the impartiality of the research reported.

\section{AUTHORS' CONTRIBUTION:}

QUA: Idea, data collection and article writing. 
NA: Review and proofreading of article.

AA: Data collection.

MG: Literature review.

$\mathrm{N}$ : Data entry and literature review.

WHS: Data analysis and review of Discussion.

\section{REFERENCES}

1. Alberti KG, Zimmet $P$, Shaw J. International diabetes federation: A consensus on type 2 diabetes prevention. Diabet Med 2007; 24:451-63.

2. Katulanda P, Constantine GR, Mahesh JG, Sheriff R, Seneviratne RD, Wijeratne S, et al. Prevalence and projections of diabetes and pre-diabetes in adults in Sri Lanka - Sri Lanka diabetes, cardiovascular study (SLDCS). Diabet Med 2008; 25:1062-9.

3. Gupta AK, McGlone M, Greenway FL, Johnson WD. Prehypertension in disease-free adults: A marker for an adverse cardiometablic risk profile. Hypertens Res 2010; 33:905-10.

4. Niroumand S, Khajedaluee $M$, Khadem-Rezaiyan $M$, Abrishami M, Juya M, Khodaee G, et al. Atherogenic index of plasma (AIP): A marker of cardiovascular disease. Med J Islam Repub Iran 2015; 29:240.

5. Nelson RH. Hyperlipidemia as a risk factor for cardiovascular disease. Prim Care 2013; 40:195-211.

6. Pacifico L, Bonci E, Andreoli G, Romaggioli S, Di Miscio R, Lombardo CV, et al. Association of serum triglyceride-to-HDL cholesterol ratio with carotid artery intima-media thickness, insulin resistance and nonalcoholic fatty liver disease in children and adolescents. Nutr Metab Cardiovasc Dis 2014; 24:737-43.

7. Kim-Dorner SJ, Deuster PA, Zeno SA, Remaley AT, Poth M. Should triglycerides and the triglycerides to high-density lipoprotein cholesterol ratio be used as surrogates for insulin resistance? Metabolism 2010; 59:299-304.

8. Ravussin E, Ryan DH. Three new perspectives on the perfect storm: What's behind the obesity epidemic? Obesity 2018; 26: 9-10.
9. Salazar MR, Carbajal HA, Espeche WG, Leiva Sisnieguez CE, Balbi'n E, Dulbecco CA, et al. Relation among the plasma triglyceride/high-density lipoprotein cholesterol concentration ratio, insulin resistance, and associated cardio-metabolic risk factors in men and women. Am J Cardiol 2012; 109:1749-53.

10. Di Bonito P, Moio N, Scilla C, Cavuto L, Sibilio G, Sanguigno E, et al. Usefulness of the high triglyceride-to-HDL cholesterol ratio to identify cardiometabolic risk factors and preclinical signs of organ damage in outpatient children. Diabetes Care 2012; 35:158-62.

11. D'Adamo E, Marcovecchio ML, Giannini C, De Giorgis T, Chiavaroli V, Chiarelli F, et al. Improved oxidative stress and cardio-metabolic status in obese prepubertal children with liver steatosis treated with lifestyle combined with vitamin E. Free Radic Res 2013; 47:146-53.

12. Cui Y, Blumenthal RS, Flaws JA, Whiteman MK, Langenberg $P$, Bachorik PS, et al. Non-high-density lipoprotein cholesterol level as a predictor of cardiovascular disease mortality. Arch Intern Med 2001; 161:1413-9.

13. Giannini C, Santoro N, Caprio S, Kim G, Lartaud D, Shaw M, et al. The triglyceride-to-HDL cholesterol ratio: Association with insulin resistance in obese youths of different ethnic backgrounds. Diabetes Care 2011; 34:1869-74.

14. Musso C, Graffigna M, Soutelo J, Honfi M, Ledesma L, Miksztowicz $\mathrm{V}$, et al. Cardiometabolic risk factors as apolipoprotein B, triglyceride/HDL-cholesterol ratio and C-reactive protein, in adolescents with and without obesity: cross-sectional study in middle class suburban children. Pediatr Diabetes 2011; 12:229-34.

15. Whitney EJ, Krasuski RA, Personius BE, Michalek JE, Maranian AM, Kolasa MW, et al. A randomized trial of a strategy for increasing high-density lipoprotein cholesterol levels: effects on progression of coronary heart disease and clinical events. Ann Intern Med 2005; 142:95-104.

16. Vega GL, Barlow CE, Grundy SM, Leonard D, DeFina LF. Triglyceride-to-high-density-lipoprotein-cholesterol ratio is an index of heart disease mortality and of incidence of type 2 diabetes mellitus in men. J Investig Med 2014; 62:345-9. 